\title{
Survival of lung adenocarcinoma patients with malignant pleural effusion
}

\author{
Shang-Gin Wu*, Chong-Jen Yu\#, Meng-Feng Tsai ${ }^{\mp}$, Wei-Yu Liao\#, Chih-Hsin Yang ${ }^{+}$, \\ I-Shiow Jan ${ }^{\S}$, Pan-Chyr Yang" and Jin-Yuan Shih"
}

\begin{abstract}
In the era of targeted therapy, the association between lung adenocarcinoma patient survival and malignant pleural effusions (MPEs) remains unclear. This study investigated the clinical characteristics, survival and epidermal growth factor receptor (EGFR) gene (EGFR) mutation status of lung adenocarcinoma patients with MPE.

From June 2005 to December 2010, consecutive pleural effusions were collected prospectively. Patient clinical characteristics, EGFR mutation status, and overall survival were analysed.

We collected MPEs from 448 patients in stage IV lung adenocarcinoma at initial diagnosis. Median overall survival for patients with MPEs at initial diagnosis and following disease progression were 14.3 months and 21.4 months, respectively $(p=0.001)$. There were $296(66.1 \%)$ patients harbouring EGFR mutations, the mutation rates among patients with an MPE at initial diagnosis and one following disease progression were $68.2 \%$ and $56.6 \%$, respectively $(p=0.044)$; the L858R mutation rate was also higher among the former $(32.6 \%$ versus $18.1 \% ; p=0.009)$. Multivariate analysis revealed that patients who: developed MPEs following disease progression, harboured EGFR mutations, and received EGFR-tyrosine kinase inhibitor therapy, had longer overall survival. Patients in stage IV lung adenocarcinoma with MPEs at initial diagnosis have shorter overall survival and higher EGFR mutation rate, especially for L858R, than patients who develop MPEs following disease progression.
\end{abstract}

KEYWORDS: EGFR mutation, EGFR-TKIs, gefitinib, lung cancer, pleural effusion

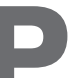
leural effusion is associated with diseases including malignancies, infections, autoimmune diseases and trauma [1]. Carcinomas of the lung, breast and lymphomas frequently cause malignant pleural effusions (MPEs). Lung adenocarcinoma is especially associated with MPEs [2], indicating advanced stage disease or disease progression.

Thoracentesis is necessary for the diagnosis and treatment of MPEs. Cancer cells in MPEs can be collected via thoracentesis, instead of through other more invasive procedures, such as biopsy or surgery [3]. Testing cancer cells for epidermal growth factor receptor (EGFR) gene (EGFR) mutation status may aid in predicting the response of EGFR-tyrosine kinase inhibitor (TKI) therapy [3]. Our previous reports have demonstrated that direct sequencing using cell-derived RNA from cell pellets of centrifuged MPEs was a sensitive detection method for EGFR mutation, without using the complicated procedures necessary to isolate cancer cells $[3,4]$. This is because inflammatory and mesothelial cells within MPEs have considerably lower EGFR expression in comparison with the overexpression of EGFR in nonsmall cell lung cancer (NSCLC) cells [4-6]. The differential expression enriches mutant EGFR from tumour cells and minimises the dilution of wild-type EGFR content from nontumour cells [4].

According to the 7th edition of the tumour-nodemetastasis (TNM) staging system, the International Association for the Study of Lung Cancer (IASLC) reclassified pleural dissemination from T4 to M1, including malignant pleural or pericardial effusions and pleural nodules [7]. Therefore, NSCLC patients with MPEs at initial diagnosis are now classified as having stage IV disease. Stage IV is further divided into M1a and M1b (distal metastasis), depending on the site of metastasis. Among stage IV patients, it is unknown whether there are differences in the demographics or survival outcomes between patients with MPEs at initial diagnosis and those who develop MPEs following disease progression.

\section{AFFILIATIONS}

*Dept of Internal Medicine, National Taiwan University Hospital Yun-Lin Branch, Yun-Lin,

${ }^{\#}$ Dept of Internal Medicine, National Taiwan University Hospital and College of Medicine, National Taiwan University, Taipei,

"Dept of Molecular Biotechnology, Dayeh University, Changhua, ${ }^{+}$Graduate Institute of Oncology, Cancer Research Centre, National Taiwan University, Taipei, and ${ }^{\text {s}}$ Dept of Laboratory Medicine, National Taiwan University Hospital, Taipei, Taiwan.

CORRESPONDENCE

J-Y. Shih

Dept of Internal Medicine National Taiwan University Hospital No. 7

Chung-Shan South Road

Taipei 100

Taiwan

E-mail: jyshih@ntu.edu.tw

Received:

May 022012

Accepted after revision:

Sept 102012

First published online:

Sept 272012 
One known predictor for survival is the presence of EGFR mutations in NSCLC patients, because they are associated with a higher response rate to EGFR-TKIs [8, 9]. However, acquired resistance develops eventually [10-13]. In approximately half of the NSCLC patients, a secondary EGFR mutation, T790M in exon 20, is detected after acquiring resistance to EGFR-TKIs $[10,11]$. Although the detection of secondary mutations after acquiring resistance to EGFR-TKIs has been well established $[10,11,14]$, whether EGFR mutations change after treatment with conventional cytotoxic chemotherapeutic agents is unknown. Furthermore, reports have found that EGFR mutation status may differ between paired primary and metastatic tumours [15-17]. Therefore, serial tissue sampling should be conducted to identify changes in EGFR mutation status.

This study investigates the clinical characteristics, survival and EGFR mutation status of lung adenocarcinoma patients with MPEs.

\section{MATERIALS AND METHODS \\ Patients and tissue procurement}

We consecutively collected pleural fluid samples from patients who received thoracentesis in the chest ultrasonography examination room of the National Taiwan University Hospital (NTUH) from June 2005 to December 2010. This study was approved by the institutional review board (IRB) of the NTUH Research Ethics Committee (IRB approval number: 983700221). Informed consents for the use of specimens in molecular studies were obtained. Cytological examinations of pleural effusions were performed. Malignant cell-positive pleural effusions were diagnosed as MPEs. Pulmonary adenocarcinoma was confirmed by pathology reports for biopsy of the primary tumours or cell blocks of MPEs with positive thyroid transcription factor-1 stains [18]. Cytology-proven MPEs caused by lung adenocarcinoma were all included and analysed for EGFR mutations. In our previous studies on EGFR mutations, materials from 225 patients were examined $[3,4]$.

Patient clinical information was recorded, including demographics, treatment regimens and treatment response. Patients who had smoked $<100$ cigarettes in their lifetimes were categorised as never-smokers [19]. Lung cancer cytology and pathology was classified according to the International Multidisciplinary Classification of Lung Adenocarcinoma criteria [18]. Disease stage was determined according to the IASLC TNM (7th Edn) staging system [7]. The unidimensional method was used to evaluate treatment response according to the Response Evaluation Criteria in Solid Tumours guidelines (version 1.1) [20]. Overall survival was defined as the period from the start date of taking first-line systemic treatment to the date of death.

\section{Collection of pleural effusion fluid and sequencing of EGFR exons 18-21}

We collected pleural fluid into heparinised tubes. A $10-\mathrm{mL}$ sample of the fluid was centrifuged at $250 \times g$ for $10 \mathrm{~min}$ at $4{ }^{\circ} \mathrm{C}$, and the total cell pellets were frozen in RNAlater (Qiagen, Hilden, Germany). The processing of samples (i.e. from sampling to freezing) took $<2 \mathrm{~h}$ as previously reported [4]. RNA was extracted from whole-cell lysate using Tri-reagent (Molecular Research Centre, Inc., Cincinnati, OH, USA) and Qiamp RNA Mini Kit (Qiagen) according to the manufacturer's protocol. Total RNA was isolated and stored at $-80^{\circ} \mathrm{C}$ until use.
Spectrophotometry was used to measure the amount of RNA extracted. The Qiagen OneStep reverse transcription (RT)-PCR kit (Qiagen) was used to obtain cDNA from extracted RNA, and exons 18-21 of EGFR were amplified. The primers and conditions of RT-PCR have been described previously [3, 4]. PCR amplicons were sequenced using ABI PRISM 3100 or 3700 (Applied Biosystems, Foster City, CA, USA) in both sense and antisense directions.

\section{EGFR mutation detection of corresponding tumour tissue by DNA sequencing}

For comparison, the use of archival tissue for EGFR gene analysis was approved by the NTUH IRB. Tumour specimens, including paraffin blocks of surgical specimens, and fine needle or bronchoscopic biopsies were obtained for mutational analysis. DNA was extracted from tumour samples. Mutational analysis for EGFR genes has been described previously [21]; some of the materials have been examined previously and reported in studies of EGFR mutations [15, 21-23].

\section{Statistical analysis}

All categorical variables were analysed using Chi-squared tests, except where sample size $n<5$ required the use of Fisher's exact test. Nonparametric Mann-Whitney U-test was used to compare the median ages of two groups. Overall survival curves were plotted using the Kaplan-Meier method and compared with log-rank test. Multivariate analysis for overall survival was performed using Cox's regression. Two-sided p-values $<0.05$ were considered significant. All analyses were performed using Statistical Package for the Social Sciences (SPSS) software (version 17.0 for Windows; SPSS Inc., Chicago, IL, USA).

\section{RESULTS}

\section{Clinical characteristics of lung adenocarcinoma patients with MPEs}

Of the 1400 pleural effusions collected, 890 MPEs were confirmed by cytological examination (online supplementary material, fig. S1). The $803 \mathrm{MPE}$ samples from lung adenocarcinomas were analysed for EGFR mutation. Because 26 samples had insufficient RNA for RT-PCR and sequencing, 777 (96.8\%) MPEs were sequenced for EGFR status. The 777 MPEs were obtained from 494 patients. The numbers and timing of MPE sampling between treatments was shown in the online supplementary table S1. The distribution of the lung cancer staging at initial diagnosis was 46 in stage I-III and 448 in stage IV. There were 713 MPEs caused by lung adenocarcinoma obtained from 448 patients with stage IV disease at initial diagnosis of NSCLC.

Of these, $365(81.5 \%)$ had MPEs at initial diagnosis, and 83 (18.5\%) developed MPEs following disease progression. There were $244(54.5 \%)$ females and $329(73.4 \%)$ never-smokers. Other clinical characteristics are presented in table 1 . Patients with MPEs at initial diagnosis were older $(p=0.002)$ and had poorer Eastern Cooperative Oncology Group performance status (ECOG PS) $(\mathrm{p}<0.001)$ than patients with MPEs after disease progression.

In addition, patients were divided into stages M1a and M1b; patient characteristics are demonstrated in table 2. Patients in stage IV M1b had poorer ECOG PS $(p=0.024)$, more lung metastasis $(p<0.001)$, and pericardial effusion $(p=0.018)$. More 
TABLE 1 Clinical characteristics of stage IV lung adenocarcinoma patients

\begin{tabular}{|c|c|c|c|c|}
\hline Total & 448 & 365 & 83 & \\
\hline Sex & & & & 0.354 \\
\hline Female & 244 & $195(53.4)$ & $49(59.0)$ & \\
\hline Male & 204 & 170 (46.6) & $34(41.0)$ & \\
\hline Smokers & 119 & 101 (27.7) & $18(21.7)$ & \\
\hline ECOG PS & & & & $<0.001$ \\
\hline $0-1$ & 354 & 276 (75.6) & $78(94.0)$ & \\
\hline $2-4$ & 94 & $89(24.4)$ & 5 (6) & \\
\hline Stage IV & & & & 0.126 \\
\hline Mutation & 296 & 249 (68.2) & 47 (56.6) & \\
\hline
\end{tabular}

Data are presented as $\mathrm{n}$, median (range) or $\mathrm{n}(\%)$, unless otherwise stated. MPE: malignant pleural effusion; ECOG PS: Eastern Cooperative Oncology Group performance status; EGFR: epidermal growth factor receptor gene. ${ }^{\#}$ : Mann-Whitney U-test.

pleural tumour seeding at initial diagnosis was noted in patients in stage IV M1a than those in stage IV M1b ( $p=0.002)$.

\section{Detection of EGFR mutations from MPEs in stage IV lung adenocarcinoma patients}

Of the total 713 samples of MPEs related to lung adenocarcinoma from 448 patients, direct sequencing using cell-derived RNA as the template were used to identify EGFR mutations. Overall, 296 (66.1\%) patients had cancer cells harbouring EGFR mutations. Females $(72.1 \%$ versus male $58.8 \% ; p=0.003)$ and never-smokers $(70.8 \%$ versus smokers $52.9 \%$; $<<0.001)$ had higher EGFR mutation rates.

Patients with MPEs at initial diagnosis had a higher rate of EGFR mutation than those with MPEs following disease progression $(68.2 \%$ versus $56.6 \% ; \mathrm{p}=0.044)$ (table 1$)$. Patients with MPEs at initial diagnosis also had a higher L858R mutation rate $(32.6 \%$ versus $18.1 \% ; \mathrm{p}=0.009)$ (table 3$)$. The proportion of presence of deletion in exon 19 (Del-19) mutation did not differ between the two groups $(26.6 \%$ versus $25.3 \%)(p=0.812)$.

\section{Overall survival of patients with stage IV lung adenocarcinoma at diagnosis}

The median overall survival for the 448 patients was 16 months. Patients with MPEs at initial diagnosis had shorter overall survival (median 14.3 months) than those with MPEs following disease progression (median 21.4 months; $p=0.001$ ) (fig. 1a). Patients whose tumour cells harboured EGFR mutations (median 17.4 months) had longer overall survival than patients whose tumour cells harboured wild-type EGFR (median 10.9 months; $\mathrm{p}=0.005$ ) (fig. $1 \mathrm{~b}$ ). In addition, patients aged $<65$ years, never-smokers, patients with better performance status (ECOG PS 0-1), patients with M1a disease status, and patients with EGFR-TKI therapy had longer overall survival (table 4).
To clarify the interaction between EGFR mutation and the timing of MPE development, overall survival of patients whose tumour cells harboured EGFR mutations and those with wildtype EGFR was plotted. Each group was further stratified by the timing of MPE development. Among patients whose tumour cells harboured EGFR mutations, those with MPEs following disease progression (median 27.1 months) had longer overall survival than those with MPEs at initial diagnosis (median 16.3 months; $\mathrm{p}=0.003$ ) (fig. 1c). In patients whose tumour cells harboured wild-type EGFR, those with MPEs following disease progression (median 16.8 months) also had longer overall survival than those with MPEs at initial diagnosis (median 8.3 months; $\mathrm{p}=0.021$ ) (fig. $1 \mathrm{~d}$ ).

EGFR mutation status and the use of EGFR-TKI therapy both had an effect on overall survival. Longest overall survival was among patients with EGFR mutations and EGFR-TKI use (18.9 months), followed sequentially by those with wild-type EGFR and who used EGFR-TKI therapy (13.8 months), those with wild-type EGFR and no EGFR-TKI therapy (7.1 months), and those with EGFR mutations and no EGFR-TKI therapy (6.3 months; $p<0.001$ ) (fig. 2). For patients with wild-type EGFR, patients without EGFR-TKI therapy were more likely to be smokers $(53.5 \%$ versus $30.3 \% ; \mathrm{p}=0.008)$, to show brain metastasis $(25.6 \%$ versus $11.9 \% ; \mathrm{p}=0.038)$ and adrenal gland metastasis $(20.9 \%$ versus $6.4 \%$; $\mathrm{p}=0.009)$ than those using EGFR-TKI therapy (online supplementary data table S2). However, the difference in survival was not statistically significant between patients with and without EGFR-TKI therapy $(\mathrm{p}=0.065)$.

To investigate the impact of the timing of MPE development, patients were stratified into stage IV M1a and M1b. Each group was further stratified by the time at which an MPE developed. In patients with stage IV M1a disease, there was no 
TABLE 2 Clinical characteristics of stage IV lung adenocarcinoma patients according to M1a and M1b division

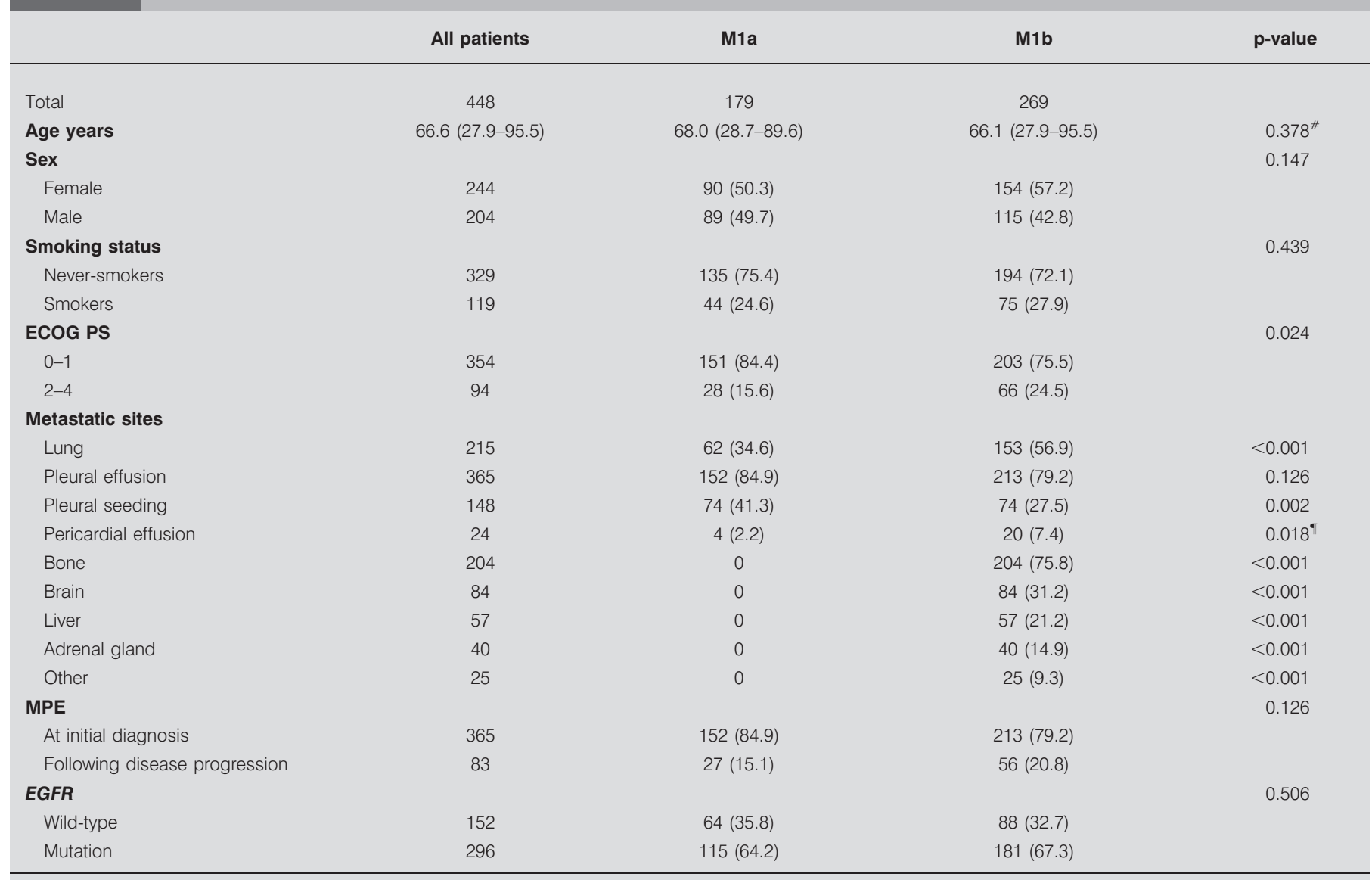

Data are presented as n, median (range) or $n$ (\%), unless otherwise stated. ECOG PS: Eastern Cooperative Oncology Group performance status; MPE: malignant pleural effusion; EFGR: epidermal growth factor receptor. "*: Mann-Whitney U-test; ": Fisher's exact test.

difference in overall survival between those with MPEs at initial diagnosis (20.9 months) and those with MPEs following disease progression ( 24 months; $\mathrm{p}=0.511$ ) (fig. 3a). In patients with stage IV M1b disease, those with MPEs at initial diagnosis (11.8 months) had shorter overall survival than those with MPEs following disease progression $(21.4$ months; $\mathrm{p}<0.001)$ (fig. 3b).
Multivariate analysis using the Cox regression model showed that having MPEs at initial diagnosis (hazard ratios 1.65; $\mathrm{p}<0.001$ ), M1b (HR 1.79; $\mathrm{p}<0.001$ ) and ECOG PS 2-4 (HR 2.22; $\mathrm{p}<0.001)$ was statistically significantly associated with shorter overall survival. Presence of EGFR mutation (HR 0.76; $\mathrm{p}=0.015$ ) and the use of EGFR-TKI therapy (HR 0.51; $\mathrm{p}<0.001$ ) were associated with longer overall survival (table 4).

TABLE 3 Differences in epidermal growth factor receptor gene (EGFR) mutation rates determined from malignant pleural effusions of stage IV adenocarcinoma at initial diagnosis and following disease progression

\begin{tabular}{|c|c|c|c|c|c|}
\hline MPE & Wild-type & Del-19 & L858R & Others & Total \\
\hline At initial diagnosis & $116(31.8)$ & $97(26.6)$ & $119(32.6)$ & $33(9.0)$ & 365 \\
\hline Following disease progression & $36(43.4)$ & $21(25.3)$ & $15(18.1)$ & $11(13.3)$ & 83 \\
\hline Total & $152(33.9)$ & $117(26.1)$ & $134(29.9)$ & $45(10.0)$ & 448 \\
\hline
\end{tabular}

Data are presented as $\mathrm{n}(\%)$ or $\mathrm{n}$. Each patient is included only once. No patients had different EGFR mutations in several malignant pleural effusion (MPE) samples, except acquired T790M EGFR mutation. Del-19: deletion in exon 19. MPEs at initial diagnosis versus MPEs after disease progression for wild-type and EGFR mutations, $p=0.044$. MPEs at initial diagnosis versus MPEs after disease progression for different mutation status, $p=0.033$. MPEs at initial diagnosis versus MPEs after disease progression for L858R versus non-L858R (including wild-type, Del-19 and other types), $p=0.009$. 

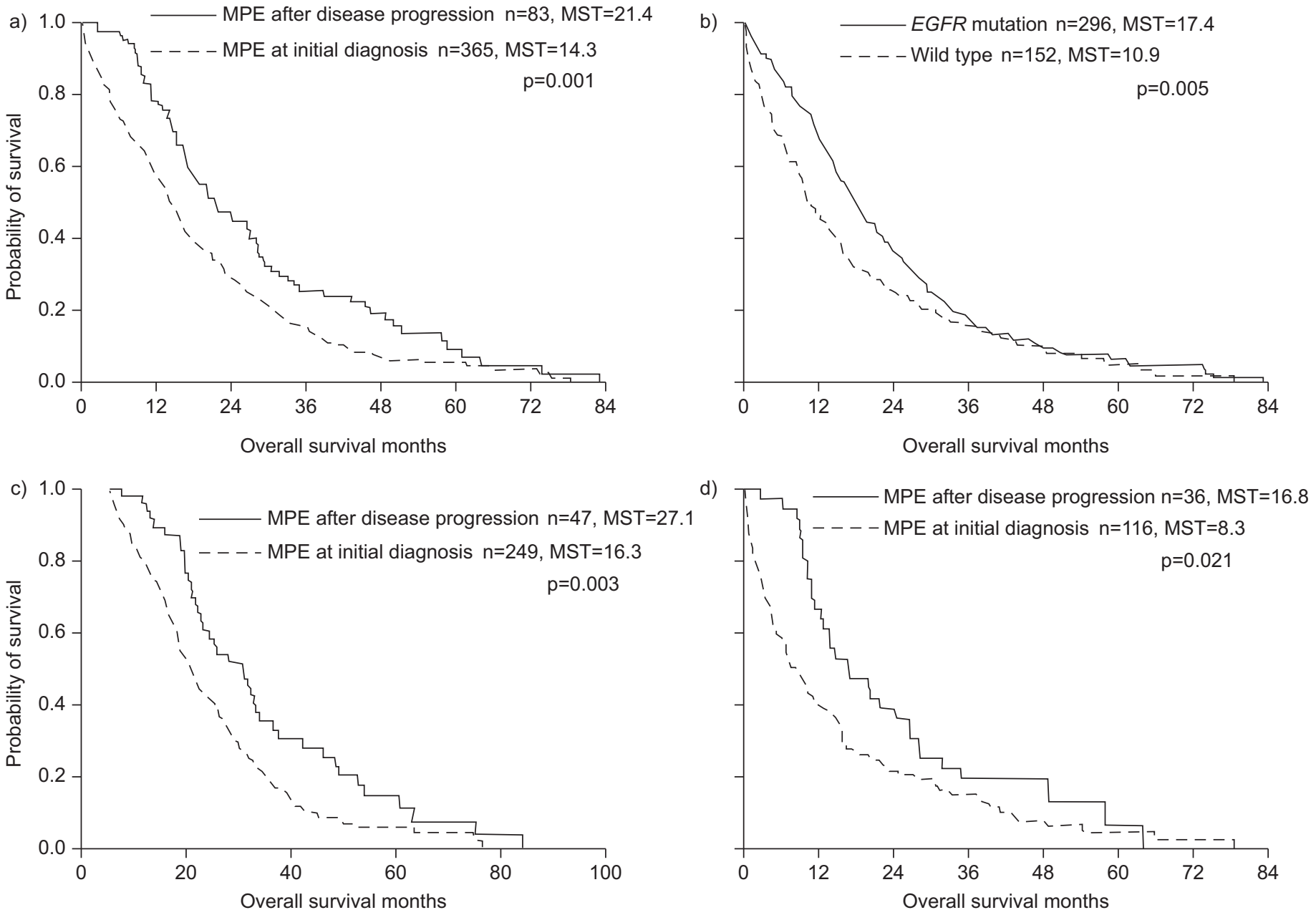

FIGURE 1. Kaplan-Meier curves of overall survival were constructed based on a) the time at which a malignant pleural effusion (MPE) developed, and b) epidermal growth factor receptor gene (EGFR) mutation sequencing results. The overall survival curves of stage IV lung adenocarcinoma patients with MPEs at initial diagnosis and those with MPEs following disease progression were plotted separately for patients with c) EGFR mutations and d) wild-type EGFR. MST: median survival time (months). pvalues were calculated using the log-rank test.

\section{No change in EGFR mutations after conventional chemotherapy}

There were 177 patients from whom two or more MPE samples (range 2-8) were obtained, and all MPE samples from the same patient were analysed for EGFR mutations. Among these, between serial samplings, 81 received chemotherapy only. The results of EGFR mutation analysis were the same among multiple MPEs from the same patient.

Of the 81 patients receiving chemotherapy only, the median duration between the first and the last MPE sampling was 204 days (range 21-1781 days). 49 patients received one regimen of chemotherapy, 20 patients received two regimens, eight patients received three regimens and four patients received four regimens between serial MPE samplings. The chemotherapy regimens used between serial MPE is shown in the online supplementary data table S3.

EGFR mutation status of the 81 patients included 35 wild-type, 19 Del-19, 18 L858R, two Del-19+T790M, two L858R+T790M, one P772_H773insYNP+H773Y, one R776H+L861Q, one L858R+ E709G, one L858R+A871E and one G719D+L861Q. No acquired mutation or change of the EGFR mutation was detected after chemotherapy, irrespective of response to the cytotoxic agents.

\section{Incidence of T790M before and after EGFR-TKI treatment}

MPEs were sampled from 317 patients before they underwent EGFR-TKI treatment. De novo T790M mutations were detected in seven $(2 \%)$ patients by direct sequencing. All seven patients had concomitant L858R mutations. Of these, six patients received EGFR-TKI (four gefitinib and two erlotinib). All six patients had progressive disease, with median progression-free survival after EGFR-TKI therapy of 1.6 months (range 0.2-2.8 months).

99 patients with acquired resistance to EGFR-TKIs had MPEs sampled after EGFR-TKI therapy. Secondary T790M mutations were detected in $48(48.5 \%)$ patients. In $23(47.9 \%)$ out of 48 patients, this secondary mutation was detected in conjunction with L858R mutations, and in 25 (52.1\%) patients, it was detected in conjunction with Del-19. Among the 48 patients with secondary T790M mutations, 16 patients underwent MPE sampling before EGFR-TKI treatment. No primary T790M mutations were detected in EGFR-TKI-naïve MPEs. 
TABLE 4 Factors affecting overall survival of stage IV lung adenocarcinoma patients with malignant pleural effusions (MPEs)

\begin{tabular}{|c|c|c|c|c|c|}
\hline & Patients & $\begin{array}{c}\text { Overall survival } \\
\text { months }\end{array}$ & $\begin{array}{c}\text { Univariate analysis } \\
\text { p-value }\end{array}$ & HR (95\% Cl) & p-value \\
\hline \multicolumn{6}{|l|}{ Sex } \\
\hline Female & 244 & 15.6 & & & \\
\hline Male & 204 & 15.5 & 0.440 & $1.00(0.77-1.30)$ & 0.974 \\
\hline$\geqslant 65$ years & 245 & 13.2 & 0.004 & $1.13(0.92-1.40)$ & 0.243 \\
\hline \multicolumn{6}{|l|}{ Smoking } \\
\hline Never-smokers & 329 & 16.5 & & & \\
\hline Smokers & 119 & 12.8 & 0.009 & $1.08(0.81-1.46)$ & 0.597 \\
\hline \multicolumn{6}{|l|}{ ECOG PS } \\
\hline M1b & 269 & 13.8 & $<0.001$ & $1.79(1.43-2.24)$ & $<0.001$ \\
\hline \multicolumn{6}{|l|}{ MPE } \\
\hline Following disease progression & 83 & 21.4 & & & \\
\hline At initial diagnosis & 365 & 14.3 & 0.001 & $1.65(1.26-2.15)$ & $<0.001$ \\
\hline \multicolumn{6}{|l|}{ EGFR } \\
\hline Wild-type & 152 & 10.9 & & & \\
\hline Mutation & 296 & 17.4 & 0.005 & $0.76(0.61-0.95)$ & 0.015 \\
\hline \multicolumn{6}{|l|}{ EGFR-TKI therapy } \\
\hline No & 80 & 6.8 & & & \\
\hline Yes & 368 & 16.8 & $<0.001$ & $0.51(0.37-0.69)$ & $<0.001$ \\
\hline
\end{tabular}

Data are presented as n, unless otherwise stated. HR: hazard ratio; 95\% Cl: 95\% confidence interval; ECOG PS: Eastern Cooperative Oncology Group performance status; EGFR: epidermal growth factor receptor; EGFR: epidermal growth factor receptor gene; TKI: tyrosine kinase inhibitor.

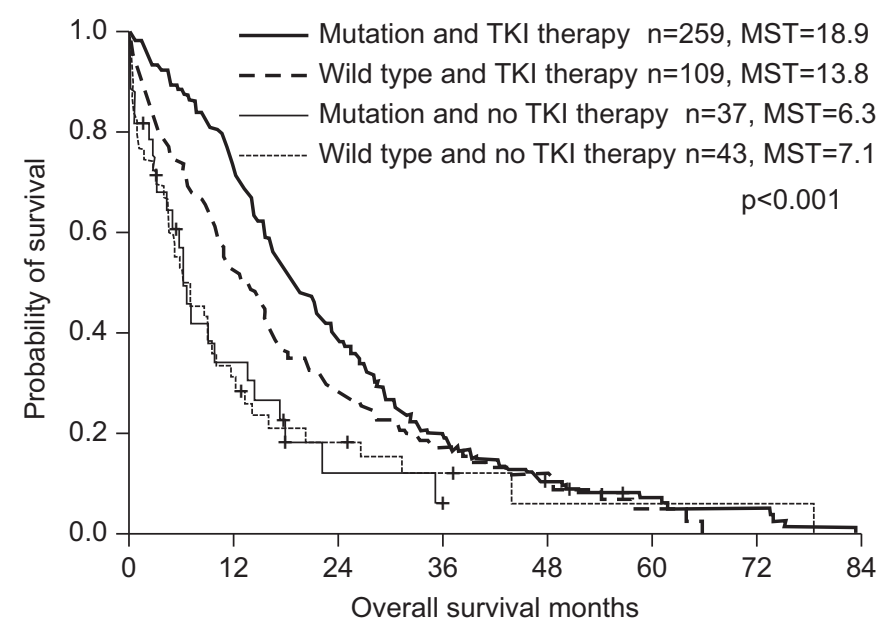

FIGURE 2. Kaplan-Meier survival curve of overall survival for stage IV lung adenocarcinoma patients with malignant pleural effusions. Epidermal growth factor receptor (EFGR) gene (EGFR) mutation-positive patients undergoing EGFR-tyrosine kinase inhibitor (TKI) therapy had longer overall survival than wild-type patients without EGFR-TKI therapy, EGFR mutation-positive patients without EGFR-TKI therapy, and wild-type patients without EGFR-TKI use $(p<0.001)$. MST: median survival time (months). p-value was calculated using the log-rank test.
Between the patients with $(n=48)$ and without $(n=51)$ secondary T790M mutations, there were no significant differences in clinical characteristics, including sex, age, smoking, ECOG PS, using gefitinib or erlotinib, stage M1a or M1b, the timing of MPE development and prior chemotherapy use (online supplementary data table S4).

\section{Correlation of the mutation analyses between the MPE and the tumour specimens}

There were 82 paired MPEs and corresponding tumour specimens. After exclusion of five tumour specimens because of poor quality DNA for EGFR mutation analysis, 62 (80.5\%) of the 77 paired samples showed concordant EGFR mutation; 15 (19.5\%) patients had discordant results between MPEs and corresponding tumour specimens (table 5).

\section{DISCUSSION}

This large prospective cohort study of MPEs derived from lung adenocarcinoma showed that the clinical characteristics of stage IV lung adenocarcinoma patients with MPEs at initial diagnosis were different from those who developed MPEs following disease progression. EGFR mutation rate, especially L858R, was higher in patients with MPEs at initial diagnosis; although all patients had stage IV disease, overall survival for 
a)

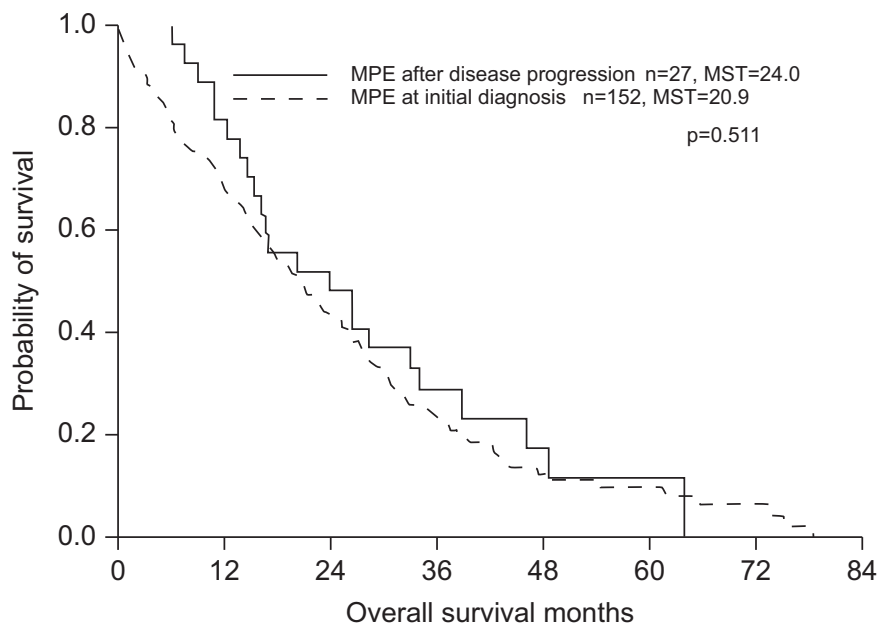

b)

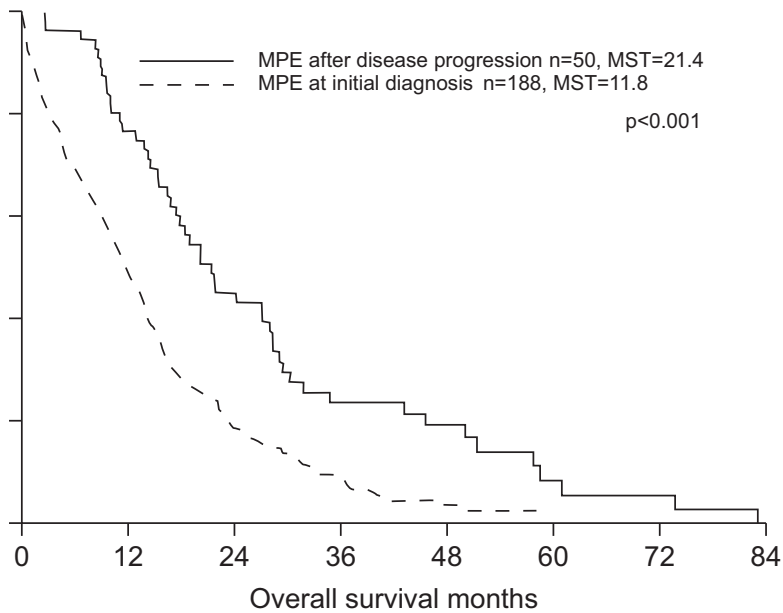

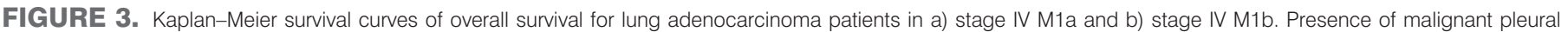
effusion (MPE) at initial diagnosis or following disease progress was plotted. MST: median survival time (months). p-values were calculated using the log-rank test.

patients with MPEs at initial diagnosis was shorter. Conventional chemotherapy did not change the mutation status or induce EGFR mutations.

Compared with tumour specimens, mutation analyses using MPEs had a high concordance rate (table 5). Thoracentesis is an easy procedure for obtaining cells to confirm the presence of MPE. Lung adenocarcinoma patients with MPEs had a higher rate of EGFR mutations [3], and a previous report also showed

\begin{tabular}{|c|c|c|c|}
\hline \multirow[t]{3}{*}{ TABLE 5} & \multicolumn{3}{|c|}{$\begin{array}{l}\text { Comparison of results of epidermal growth factor } \\
\text { receptor gene (EGFR) mutation analysis between } \\
\text { malignant pleural effusions (MPE) and tumour } \\
\text { specimens }\end{array}$} \\
\hline & \multicolumn{2}{|c|}{ EGFR mutation analysis } & \multirow[t]{2}{*}{ Patients } \\
\hline & MPE & Tumour specimens & \\
\hline \multirow[t]{9}{*}{ Concordance } & & & $62(80.5)$ \\
\hline & Wild-type & Wild-type & $24(31.2)$ \\
\hline & Del-19 & Del-19 & $12(15.6)$ \\
\hline & L858R & L858R & $18(23.4)$ \\
\hline & Insertion in exon 20 & Insertion in exon 20 & $4(5.2)$ \\
\hline & G719A+S720F & G719A+S720F & $1(1.3)$ \\
\hline & G719A+T790M & G719A+T790M & $1(1.3)$ \\
\hline & L858R+E709G & L858R+E709G & $1(1.3)$ \\
\hline & L858R+A871E & L858R+A871E & $1(1.3)$ \\
\hline \multirow[t]{7}{*}{ Discordance } & & & $15(19.5)$ \\
\hline & Del-19 & Wild-type & $7(9.1)$ \\
\hline & L858R & Wild-type & $4(5.2)$ \\
\hline & L861Q+G719S & Wild-type & $1(1.3)$ \\
\hline & L861Q+E746G & Wild-type & $1(1.3)$ \\
\hline & Wild-type & Del-19 & $1(1.3)$ \\
\hline & Wild-type & L858R & $1(1.3)$ \\
\hline Total & & & $77(100)$ \\
\hline
\end{tabular}

Data are presented as n (\%), unless otherwise stated. that direct sequencing using cell-derived RNA was very sensitive and favourable for EGFR mutation detection in lung adenocarcinoma MPEs [4]. If tumour specimens were used, less than half of the patients would have adequate specimens to obtain a molecular profile $[9,24]$. In addition, because DNA from cancer cells makes only a small fraction of total DNA, using specimens with high proportion of non-neoplastic cells, such as biopsy samples, lowers the sensitivity for mutation analysis [25]. Furthermore, patients are more likely to accept repeated thoracentesis rather than re-biopsy of the primary tumour to detect molecular changes, making thoracentesis an important procedure in monitoring disease status for personalising treatment for lung cancer.

Although the presence of an MPE is a significant prognostic factor for advanced-stage NSCLC patients [26, 27], no studies on the effects of the time at which an MPE developed on the overall survival of a patient have been conducted. In this study, stage IV lung adenocarcinoma patients who developed MPEs following disease progression showed longer overall survival than those with MPEs at initial diagnosis. This effect is observed in patients with EGFR mutations and wild-type EGFR. However, the difference was only statistically significant in patients with distal metastasis (M1b). For patients without distal metastasis (M1a) there was no difference. In the present study, patients with MPEs at initial diagnosis of stage IV lung adenocarcinoma had overall survival of 16.2 months, which was longer than the previously reported 6.5-8 months [27-29]. The longer overall survival in the present study may result from four possible reasons. First, only patients of adenocarcinoma, which had higher EGFR mutations, were included in this study, and those with MPEs had a higher EGFR mutation rate [3]. Most patients of the previous studies were from Western countries, where the EGFR mutation rate is low. Secondly, patients in previous studies were enrolled before the approval of EGFR-TKI therapy. In this study, a high proportion 368 (82.1\%) out of 448 patients received EGFR-TKI treatment. TAKANO et al. [30] showed that patients with lung cancer treated after the approval of gefitinib had longer overall survival than those treated before gefitinib 
approval. Thirdly, overall survival in phase III trials of EGFRTKI in Asians ranged from 18.8 months to 22.3 months [31, 32]. Ethnic difference might be responsible for the difference in overall survival. Finally, a selection bias of patient collection could have been introduced into the study. The patients may have a better performance status because they were able to have thoracentesis in the chest ultrasonography examination room.

We previously showed that patients with MPEs associated with lung adenocarcinoma had a higher EGFR mutation rate than that observed in surgically resected specimens [3]. With more patients enrolled in the present study, we found that the overall EGFR mutation rate was similar to that in our previous report [3]. However, patients with MPEs at initial diagnosis had a significantly higher rate of mutation than patients with MPEs after disease progression; in particular, the L858R mutation rate was significantly higher. In our previous study, a higher L858R mutation rate was also observed in MPEs from lung adenocarcinoma than in surgically resected samples. From these observations, it has been postulated that EGFR mutation is an early event in the pathogenesis of lung adenocarcinoma [33]. L858R may play a role in the development of MPE among lung adenocarcinoma patients. Further studies are necessary to clarify the relationship between EGFR mutations and metastasis of lung adenocarcinoma.

Gene mutation could result in acquired resistance to target therapy drugs. In chronic myeloid leukaemia and gastrointestinal stromal tumours, T315I, which causes a protein structural change, is considered to be one of the most common imatinib resistance mutations in $B C R-A B L$ [34-36]. For breast cancer, XIA et al. [37] found that acquired resistance to lapatinib, a potent ErbB2 TKI, occurred when cell survival regulation is changed to depend on both oestrogen receptor and ErbB2. All these acquired resistance-associated mutations developed after patients received target therapy. For NSCLC, the mutations T790M, D761Y and L747S are all considered to be associated with acquired resistance to EGFR-TKIs [10, 38, 39]. For cytotoxic drugs, studies have shown cancers to develop drug resistance. After acquiring resistance to cytotoxic agents, there were several changes in cancer cells, including gene amplification, gene rearrangements, epigenetic changes, and microRNA changes [40]. Apart from one conference poster demonstrating that chemotherapy could influence EGFR mutation status [41], no drug resistance through gene mutation induced by cytotoxic drugs has been documented. Comparing paired cancer cells before and after chemotherapy from MPEs, we found that cytotoxic chemotherapy did not alter EGFR mutation status. This implies that after chemotherapy, EGFR mutant lung cancer cells preserve the sensitive mutations and, therefore, cell response to EGFR-TKI. This finding is consistent with previous reports showing that response rate and progression-free survival in patients with sensitive EGFR mutations were similar whether EGFR-TKI was used as first-line or second-line therapy [22, 42]. A large prospective study would further clarify this effect.

The secondary T790M mutation was reported to occur in $50 \%$ of patients with EGFR mutations after EGFR-TKI treatment $[11,43,44]$. In the present study, the secondary T790M mutation rate was $48.5 \%$, which was consistent with the previously reported rate. However, we still cannot predict who will develop secondary T790M mutations after acquired resistance to EGFRTKIs based on clinical features.
For patients with wild-type EGFR, although our study showed patients undergoing EGFR-TKI therapy had longer survival than those without EGFR-TKI therapy, it did not reach statistical significance $(p=0.065)$. The difference may result from the following reasons. First, the BR.21 study showed a survival benefit of erlotinib in NSCLC patients in all statuses, including patients with wild-type EGFR [45]. A phase-II trial showed that the overall survival after erlotinib treatment was 9.2 months in patients with wild-type EGFR who had received chemotherapy previously [46]. The present study showed that the median overall survival after EGFR-TKI treatment was 8.3 months (95\% CI 4.3-12.3 months) in patients with wildtype EGFR, which is compatible with previous reports [46]. Secondly, in the present study, patients with wild-type EGFR and no EGFR-TKI therapy were more likely to be smokers and to have brain metastasis and adrenal gland metastasis than those with wild-type EGFR and EGFR-TKI therapy. Finally, the response rate of EGFR-TKI therapy in patients with wild-type EGFR was $10-20 \%$ in previous reports [8, 12, 32, 47, 48].

The present study has some limitations. First, patients enrolled in the present study were all Taiwanese, among whom a high EGFR mutation rate, even among smokers, has been documented [49]. Using resected lung adenocarcinoma samples, HUANG et al. [50] also showed that the EGFR mutation rate was not associated with sex or smoking status in Taiwan. Because of the ethnic uniformity of the study population, the findings of the present study might not be general to other racial or ethnic groups. Secondly, some patients with tumours harbouring EGFR mutations did not receive EGFR-TKI treatment in the present study because EGFR mutation analysis was not routinely performed in clinical practice at the time of sample collection.

In conclusion, stage IV lung adenocarcinoma patients with MPEs at initial diagnosis have a higher EGFR mutation rate, especially for L858R, and shorter overall survival than patients who develop MPEs following disease progression. EGFR mutations and EGFR-TKI therapy are associated with longer overall survival in lung adenocarcinoma patients with MPEs.

\section{SUPPORT STATEMENT}

This study was supported by grants 98-2314-B-002-117-MY3, 982628-B-002-087-MY3 (National Science Council, Taiwan), DOH100TD-PB-111-TM001 (Department of Health, Executive Yuan, Taiwan) and 99C101-101, 100C101-101 and 10R71601-3 (National Taiwan University, Taiwan).

\section{STATEMENT OF INTEREST}

None declared.

\section{ACKNOWLEDGMENTS}

The authors would like to thank the Department of Medical Research at the National Taiwan University Hospital for providing the laboratory facility.

\section{REFERENCES}

1 Light RW. Clinical practice. Pleural effusion. N Engl J Med 2002; 346: 1971-1977.

2 Wozniak A, Gadgeel S, eds. Clinical presentation of non-small cell carcinoma of the lung. In: Pass HI, Johnson DH, eds. Lung Cancer: Principles and Practice. 3rd Edn. Philadelphia, Lippincott Williams \& Wilkins, 2005; pp. 291-303. 
$3 \mathrm{Wu}$ SG, Gow CH, Yu CJ, et al. Frequent epidermal growth factor receptor gene mutations in malignant pleural effusion of lung adenocarcinoma. Eur Respir J 2008; 32: 924-930.

4 Tsai TH, Su KY, Wu SG, et al. RNA is favourable for analysing EGFR mutations in malignant pleural effusion of lung cancer. Eur Respir J 2012; 39: 677-684

5 Hirsch FR, Varella-Garcia M, Bunn PA Jr, et al. Epidermal growth factor receptor in non-small-cell lung carcinomas: correlation between gene copy number and protein expression and impact on prognosis. J Clin Oncol 2003; 21: 3798-3807.

6 Brabender J, Danenberg KD, Metzger R, et al. Epidermal growth factor receptor and HER2-neu mRNA expression in non-small cell lung cancer is correlated with survival. Clin Cancer Res 2001; 7: $1850-1855$.

7 Goldstraw P, Crowley J, Chansky K, et al. The IASLC Lung Cancer Staging Project: proposals for the revision of the TNM stage groupings in the forthcoming (seventh) edition of the TNM Classification of malignant tumours. J Thorac Oncol 2007; 2: 706-714.

8 Lynch TJ, Bell DW, Sordella R, et al. Activating mutations in the epidermal growth factor receptor underlying responsiveness of non-small-cell lung cancer to gefitinib. N Engl J Med 2004; 350: 2129-2139.

9 Mok TS, Wu YL, Thongprasert S, et al. Gefitinib or carboplatinpaclitaxel in pulmonary adenocarcinoma. N Engl J Med 2009; 361: 947-957.

10 Kobayashi S, Boggon TJ, Dayaram T, et al. EGFR mutation and resistance of non-small-cell lung cancer to gefitinib. $N$ Engl J Med 2005; 352: 786-792.

11 Pao W, Miller VA, Politi KA, et al. Acquired resistance of lung adenocarcinomas to gefitinib or erlotinib is associated with a second mutation in the EGFR kinase domain. PLoS Med 2005; 2: e73.

12 Sharma SV, Bell DW, Settleman J, et al. Epidermal growth factor receptor mutations in lung cancer. Nat Rev Cancer 2007; 7: 169-181.

13 Jackman D, Pao W, Riely GJ, et al. Clinical definition of acquired resistance to epidermal growth factor receptor tyrosine kinase inhibitors in non-small-cell lung cancer. J Clin Oncol 2010; 28: 357-360

14 Inukai M, Toyooka S, Ito S, et al. Presence of epidermal growth factor receptor gene T790M mutation as a minor clone in nonsmall cell lung cancer. Cancer Res 2006; 66: 7854-7858.

15 Gow $\mathrm{CH}$, Chang YL, Hsu YC, et al. Comparison of epidermal growth factor receptor mutations between primary and corresponding metastatic tumors in tyrosine kinase inhibitor-naive non-small-cell lung cancer. Ann Oncol 2009; 20: 696-702.

16 Park S, Holmes-Tisch AJ, Cho EY, et al. Discordance of molecular biomarkers associated with epidermal growth factor receptor pathway between primary tumors and lymph node metastasis in non-small cell lung cancer. J Thorac Oncol 2009; 4: 809-815.

17 Schmid K, Oehl N, Wrba F, et al. EGFR/KRAS / BRAF mutations in primary lung adenocarcinomas and corresponding locoregional lymph node metastases. Clin Cancer Res 2009; 15: 4554-4560.

18 Travis WD, Brambilla E, Noguchi M, et al. International Association for the Study of Lung Cancer/American Thoracic Society/ European Respiratory Society international multidisciplinary classification of lung adenocarcinoma. J Thorac Oncol 2011; 6: 244-285.

19 Centers for Disease Control and Prevention. Cigarette smoking among adults - United States, 2006. MMWR Morb Mortal Wkly Rep 2007; 56: 1157-1161.

20 Therasse P, Arbuck SG, Eisenhauer EA, et al. New guidelines to evaluate the response to treatment in solid tumors. European Organization for Research and Treatment of Cancer, National Cancer Institute of the United States, National Cancer Institute of Canada. J Natl Cancer Inst 2000; 92: 205-216.

21 Shih JY, Gow CH, Yu CJ, et al. Epidermal growth factor receptor mutations in needle biopsy/aspiration samples predict response to gefitinib therapy and survival of patients with advanced nonsmall cell lung cancer. Int J Cancer 2006; 118: 963-969.

$22 \mathrm{Wu}$ JY, Yu CJ, Yang CH, et al. First- or second-line therapy with gefitinib produces equal survival in non-small cell lung cancer. Am J Respir Crit Care Med 2008; 178: 847-853.

$23 \mathrm{Wu}$ JY, Wu SG, Yang CH, et al. Lung cancer with epidermal growth factor receptor exon 20 mutations is associated with poor gefitinib treatment response. Clin Cancer Res 2008; 14: 4877-4882.

24 Pao W, Chmielecki J. Rational, biologically based treatment of EGFR-mutant non-small-cell lung cancer. Nat Rev Cancer 2010; 10: 760-774.

25 Pao W, Ladanyi M. Epidermal growth factor receptor mutation testing in lung cancer: searching for the ideal method. Clin Cancer Res 2007; 13: 4954-4955.

26 Naito $\mathrm{T}$, Satoh $\mathrm{H}$, Ishikawa $\mathrm{H}$, et al. Pleural effusion as a significant prognostic factor in non-small cell lung cancer. Anticancer Res 1997; 17: 4743-4746.

27 Sugiura S, Ando Y, Minami H, et al. Prognostic value of pleural effusion in patients with non-small cell lung cancer. Clin Cancer Res 1997; 3: 47-50.

28 Alon BN, Anson BL. Pleural effusion in patients with non-small cell carcinoma - stage IV and not T4. Lung Cancer 2007; 57: 123.

29 Rami-Porta R, Ball D, Crowley J, et al. The IASLC Lung Cancer Staging Project: proposals for the revision of the $\mathrm{T}$ descriptors in the forthcoming (seventh) edition of the TNM classification for lung cancer. J Thorac Oncol 2007; 2: 593-602.

30 Takano T, Fukui T, Ohe Y, et al. EGFR mutations predict survival benefit from gefitinib in patients with advanced lung adenocarcinoma: a historical comparison of patients treated before and after gefitinib approval in Japan. J Clin Oncol 2008; 26: 5589-5595.

31 Fukuoka M, Wu YL, Thongprasert S, et al. Biomarker analyses and final overall survival results from a phase III, randomized, openlabel, first-line study of gefitinib versus carboplatin/paclitaxel in clinically selected patients with advanced non-small-cell lung cancer in Asia (IPASS). J Clin Oncol 2011; 29: 2866-2874.

32 Han JY, Park K, Kim SW, et al. First-SIGNAL: first-line singleagent iressa versus gemcitabine and cisplatin trial in neversmokers with adenocarcinoma of the lung. J Clin Oncol 2012; 30: 1122-1128.

33 Tang $\mathrm{X}$, Shigematsu $\mathrm{H}$, Bekele BN, et al. EGFR tyrosine kinase domain mutations are detected in histologically normal respiratory epithelium in lung cancer patients. Cancer Res 2005; 65: 7568-7572.

34 Gorre ME, Mohammed M, Ellwood K, et al. Clinical resistance to STI-571 cancer therapy caused by $B C R-A B L$ gene mutation or amplification. Science 2001; 293: 876-880.

35 Chen LL, Trent JC, Wu EF, et al. A missense mutation in KIT kinase domain 1 correlates with imatinib resistance in gastrointestinal stromal tumors. Cancer Res 2004; 64: 5913-5919.

36 Azam M, Latek RR, Daley GQ. Mechanisms of autoinhibition and STI-571/imatinib resistance revealed by mutagenesis of $B C R-A B L$. Cell 2003; 112: 831-843.

37 Xia W, Bacus S, Hegde P, et al. A model of acquired autoresistance to a potent ErbB2 tyrosine kinase inhibitor and a therapeutic strategy to prevent its onset in breast cancer. Proc Natl Acad Sci USA 2006; 103: 7795-7800.

38 Balak MN, Gong Y, Riely GJ, et al. Novel D761Y and common secondary T790M mutations in epidermal growth factor receptormutant lung adenocarcinomas with acquired resistance to kinase inhibitors. Clin Cancer Res 2006; 12: 6494-6501.

39 Costa DB, Schumer ST, Tenen DG, et al. Differential responses to erlotinib in epidermal growth factor receptor (EGFR)-mutated lung cancers with acquired resistance to gefitinib carrying the L747S or T790M secondary mutations. J Clin Oncol 2008; 26: 1182-1184.

40 Fojo T. Multiple paths to a drug resistance phenotype: mutations, translocations, deletions and amplification of coding genes or promoter regions, epigenetic changes and microRNAs. Drug Resist Updat 2007; 10: 59-67. 
41 Wang J, Chen $\mathrm{K}$, Bai $\mathrm{H}$, et al. Chemotherapy influence EGFR mutation status for Chinese patients with regionally advanced non-small cell lung cancer. J Clin Oncol 2011; 29: Suppl. A7031.

42 Rosell R, Moran T, Queralt C, et al. Screening for epidermal growth factor receptor mutations in lung cancer. N Engl J Med 2009; 361: 958-967.

43 Kosaka T, Yatabe Y, Endoh H, et al. Analysis of epidermal growth factor receptor gene mutation in patients with non-small cell lung cancer and acquired resistance to gefitinib. Clin Cancer Res 2006; 12: 5764-5769.

44 Bean J, Riely GJ, Balak M, et al. Acquired resistance to epidermal growth factor receptor kinase inhibitors associated with a novel T854A mutation in a patient with EGFR-mutant lung adenocarcinoma. Clin Cancer Res 2008; 14: 7519-7525.

45 Tsao MS, Sakurada A, Cutz JC, et al. Erlotinib in lung cancer molecular and clinical predictors of outcome. N Engl J Med 2005; 353: 133-144.

46 Yoshioka H, Hotta K, Kiura K, et al. A phase II trial of erlotinib monotherapy in pretreated patients with advanced non-small cell lung cancer who do not possess active EGFR mutations: Okayama Lung Cancer Study Group trial 0705. J Thorac Oncol 2010; 5: 99-104.

47 Mitsudomi T, Kosaka T, Endoh H, et al. Mutations of the epidermal growth factor receptor gene predict prolonged survival after gefitinib treatment in patients with non-small-cell lung cancer with postoperative recurrence. J Clin Oncol 2005; 23: 2513-2520.

48 Taguchi F, Solomon B, Gregorc V, et al. Mass spectrometry to classify non-small-cell lung cancer patients for clinical outcome after treatment with epidermal growth factor receptor tyrosine kinase inhibitors: a multicohort cross-institutional study. J Natl Cancer Inst 2007; 99: 838-846.

49 Yang PC, Yuankai S, Joseph Au S, et al. Molecular epidemiological prospective study of EGFR mutations from Asian patients (pts) with advanced lung adenocarcinoma (PIONEER). J Clin Oncol 2012; 30: Suppl. A1534.

50 Huang SF, Liu HP, Li LH, et al. High frequency of epidermal growth factor receptor mutations with complex patterns in non-small cell lung cancers related to gefitinib responsiveness in Taiwan. Clin Cancer Res 2004; 10: 8195-8203. 